\title{
Atomistic-continuum hybrid analysis of dislocation behavior in spinodally decomposed Fe-Cr alloys
}

\author{
Akiyuki Takahashi ${ }^{1}$ and Motoyasu Kanazawa $^{1}$ \\ ${ }^{1}$ Department of Mechanical Engineering, Faculty of Science and Technology, Tokyo University of Science, 2641 Yamazaki, \\ Nodashi, Chiba, 278-8510, Japan
}

\begin{abstract}
In this study, we first present the molecular dynamics (MD) simulation of dislocation behavior in a spinodally decomposed $\mathrm{Fe}-\mathrm{Cr}$ alloy. The MD simulation is used for exploring the nature of the interaction between a dislocation and the spinodal decomposition without any specific assumptions. In order to classify the interaction mechanism, dislocation dynamics (DD) simulations of the interaction between a dislocation and the spinodal decomposition are performed. In the simulations, we controlled the interaction mechanism by adding and removing the atomistic mechanism. The simulation results clearly illustrate that the atomistic mechanism can be negligible in determining the critical resolved shear stress (CRSS) of spinodally decomposed $\mathrm{Fe}-\mathrm{Cr}$ alloys, and the internal stress generated by the lattice constant mismatch is a dominant mechanism. These findings are very useful for simplifying the analysis of the mechanism of material strength change due to the spinodal decomposition. Particularly in the analysis using the DD simulations, the required computational effort for simulating the dislocation behavior is greatly reduced by taking into account only the internal stress without the atomistic dislocation core influence.
\end{abstract}

\section{Introduction}

Duplex stainless steels consisting of ferrite and austenite phases have a high material strength, particularly the corrosion resistance, and are used as a material of primary coolant pipes in nuclear power plants. When the material is aged at temperatures in a range from 300 to $500^{\circ} \mathrm{C}$, spinodal decomposition occurs in the ferrite phase, which causes an ultrafine phase separation mixing Fe-rich and $\mathrm{Cr}$-rich phases. The phase separation leads to a material embrittlement and material strength change, and therefore, it is very important to understand the influence of the phase separation on the material strength for ensuring the reliability and integrity of structures. In order to investigate the material strength degeneration mechanism, an equation for the internal stress distribution arisen from the phase separation has been derived [1]. The equation can be used as a fundamental tool for investigating the influence of phase separation on the micro-scale material deformation mechanism, such as dislocation behavior. Kato conducted extensive theoretical studies on the interaction of the internal stress and dislocation and on the influence on the critical resolved shear stress (CRSS), which is a shear stress necessary for dislocations to initiate their motion in materials [2]. The information obtained by the studies is variable in clarifying the dislocation behavior in the internal stress field, and however, due to the complexity and limitation of the theoretical approach to the problem, the information is limited for dislocations with a simple shape, even though the dislocation shape must be changed a lot by the interaction with the internal stress field. In addition, the influence of the spinodal decomposition on the material strength must be controlled by not only the internal stress field, and also the other type of elements associated with atomistic chemical energies. Thus, in order to fully understand the detailed mechanism of the material strength degeneration due to the spinodal decomposition, the understanding must cover very wide range from atomistic to continuum.

Owing to a remarkable development of dislocation dynamics (DD) simulation methodology, collective behavior and complex interactions of dislocations can be simulated and calculated using computers [3-5]. Up till now, the DD method has been successfully applied to various plasticity problems of metals and alloys. Takahashi and Ghoniem have developed a dislocation dynamics-based computational method for dislocationprecipitate interaction problems, and investigated the interaction of dislocations with precipitates in terms of elasticity [6]. Furthermore, they developed a hybrid atomistic-continuum method for investigation of dislocation cores [7-8]. The method provides us with a new opportunity to study the dislocation dynamics

\footnotetext{
" Corresponding author: takahash@rs.noda.tus.ac.jp
} 
coupled with the atomistic influence of dislocation cores. Thus, the type of simulation method could be a powerful tool for investigating the material strength degeneration mechanism due to the spinodal decomposition involving both the continuum and atomistic mechanisms.

In this study, we first present the molecular dynamics (MD) simulation of dislocation behavior in a spinodally decomposed $\mathrm{Fe}-\mathrm{Cr}$ alloy. The MD simulation is used for exploring the nature of the interaction between a dislocation and the spinodal decomposition without any specific assumptions. In order to classify the interaction mechanism, DD simulations of the interaction between a dislocation and the spinodal decomposition are performed. In the simulations, we controlled the interaction mechanism by adding and removing the atomistic mechanism, say the chemical influence. In order to deal with the atomistic mechanism, we employed the discretized Peierls-Nabarro model in conjunction with the generalized stacking fault energy (GSFE). Finally, we discuss the importance of the continuum and atomistic mechanism on the material strength change due to the spinodal decomposition.

\section{Computational methods}

\subsection{Molecular dynamics method}

The interaction between a dislocation and the spinodal decomposition is first examined using the MD simulations. In the MD simulations, an embedded atom method (EAM) type of interatomic potential developed for $\mathrm{Fe}-\mathrm{Cr}$ alloy is used [9]. Figure 1 shows the simulation volume for the MD simulations. The size of simulation volume is $14.71 \times 11.25 \times 8.52 \mathrm{~nm}$, and the $\mathrm{x}, \mathrm{y}$ and $\mathrm{z}$ axes coincide with crystal orientations of [111], [110] and [112], respectively. The average $\mathrm{Cr}$ atom concentration is $20 \%$. The spinodal decomposition is made in the simulation volume by distributing the $\mathrm{Cr}$ atoms using the cosine function. Figure 1(b) shows the distribution of $\mathrm{Cr}$ atoms in $\mathrm{Fe}$ matrix. It can be seen that $\mathrm{Cr}$ atoms are well modulated using the cosine function. The magnitude of cosine function, which is equal to the magnitude of fluctuation of $\mathrm{Cr}$ atom concentration, is changed in a range from 0 to $20 \%$ in order to see the influence on the dislocation behavior. As a boundary condition, the atoms in the upper and lower layer in the y-direction cannot move in the y-direction, and are enforced to move by a prescribed displacement in the $\mathrm{x}$-direction. The simulation volume is then relaxed using the fast inertia relaxation engine (FIRE) algorithm [10]. Using the procedure, the stress-strain behavior of the simulation volume is obtained. The critical resolved shear stress (CRSS), which is a necessary stress for dislocations to initiate their motion, is determined as the peak shear stress in the stress-strain behavior. (a)

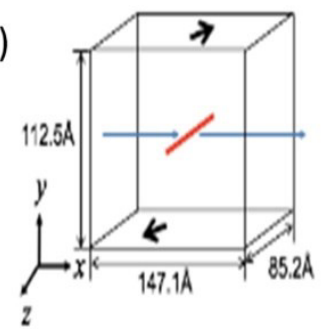

(b)

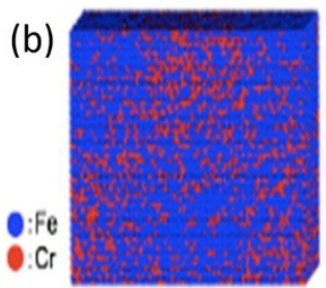

Fig. 1 MD simulation volume

\subsection{Dislocation dynamics method}

Although, the MD simulations can provide us with the information on the dislocation behavior and CRSS, it is impossible to classify the elementary mechanisms controlling the interaction. In order to clarify what mechanism is dominantly working in the interaction, we perform the DD simulation with the same simulation condition that we use in the MD simulations. In the DD simulation, dislocations are discretized into a number of line (dislocation) segments. The force acting on each dislocation segment is calculated based on the elasticity of dislocations. In this work, we will take into account the influence of internal stress (elastic interaction) and the atomistic mechanism (dislocation core influence). The equation of internal stress produced by the spinodally decomposition is derived by Cahn [1], and thus, the equation is utilized in the force calculation in the DD simulations. On the other hand, in order to account for the atomistic influence in the DD simulation, the discretized Peierls-Nabarro (PN) model is employed [7]. In the model, dislocation core structure is described with a collection of discrete infinitesimal dislocations. Within the dislocation core, the infinitesimal dislocations experience both the lattice restoring force and the elastic interaction between the infinitesimal dislocations. The lattice restoring force results from the stacking fault energy expanding in the dislocation core, which can be calculated using the GSFE. The GSFE can be obtained using MD calculations.

\section{Simulation results}

\subsection{Molecular dynamics simulation results}

Figures 2 and 3 show the stress-strain behavior and the snapshots of the MD simulation with the magnitude of fluctuation of $0 \%$. In this paper, the dislocation shape is found using the common neighbor analysis (CNA) technique [11], and only the atoms extracted by the CNA are displayed in the figure. The color shows the internal stress distribution on the slip plane of dislocation calculated using the internal stress equation. 


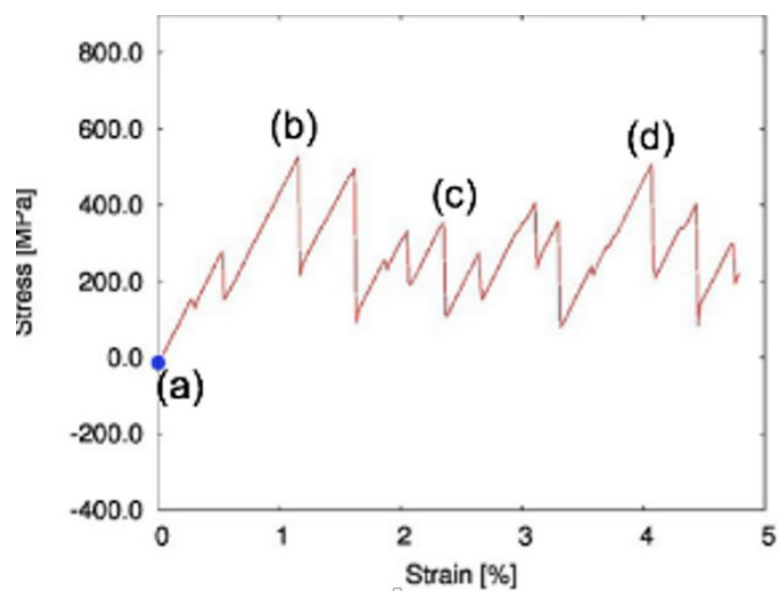

Fig. 2 Stress-strain behavior with the magnitude of $\mathrm{Cr}$ atom fluctuation of $0 \%$ calculated by $\mathrm{MD}$ simulations. The corresponding snapshots in Fig. 3 are indicated with a label of (a)-(d)

The stress component is along the direction of the Burgers vector of the dislocation on the slip plane. Based on the continuum elasticity of dislocations, the shear stress shown by the blue color gives a resistance force to the dislocation motion, while the shear stress shown by the red color gives an attractive force to the dislocation motion. It can be seen that, in this case, the stress distribution is in a striped pattern. As shown in Fig. 3, the dislocation is affected by the distribution of $\mathrm{Cr}$ atoms. Therefore, the shape of dislocation is not straight. In the stress-strain behavior shown in Fig 2, the stress-strain behavior has a zig-zag shape. The shape must result from the local $\mathrm{Cr}$ atom locations near the dislocation. Then, we found that the CRSS is about $450 \mathrm{MPa}$.

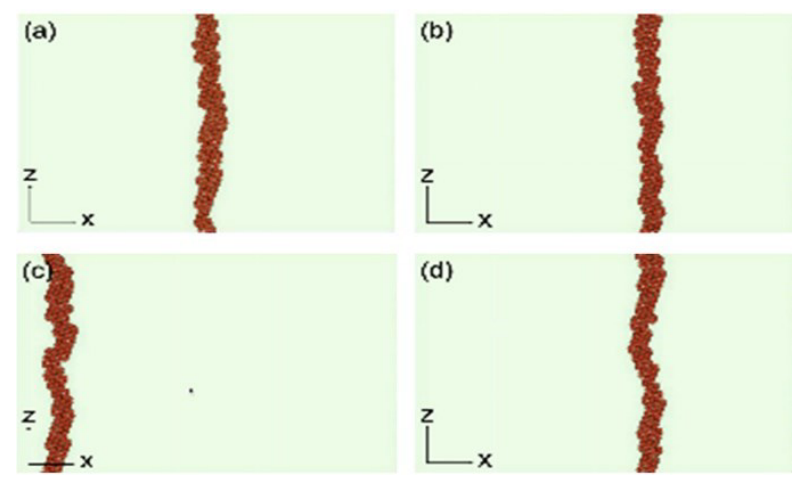

Fig. 3 Dislocation behavior in the MD simulation with a magnitude of $\mathrm{Cr}$ atom fluctuation of $0 \%$. Red particles show the atoms around the dislocation core.

On the other hand, when the magnitude of fluctuation is $20 \%$, the stress-strain behavior and the snapshots of the MD simulation are shown in Fig 4 and 5 respectively. In Fig. 5, the dislocation is first absorbed by the shear stress shown by the red color. After then, the dislocation comes to the shear stress shown by the blue color. However, in front of the shear stress, the dislocation stops, because the shear stress gives a resistance force to the dislocation. When the applied shear stress becomes large enough, the dislocation can pass the shear stress region. In the stress-strain behavior shown in Fig. 4, the stress drops down at the shear strain of $0.5 \%$. At the shear stress, the dislocation is absorbed by the shear stress shown by red color. At the shear stress of $3.2 \%$, the stress shows the peak value of $800 \mathrm{MPa}$, which is the CRSS.

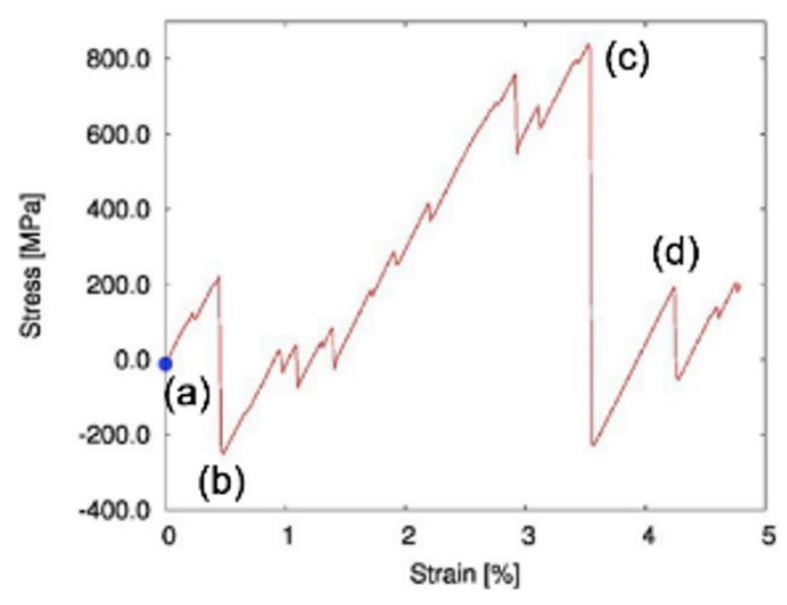

Fig. 4 Stress-strain behavior with the magnitude of $\mathrm{Cr}$ atom fluctuation of $20 \%$ calculated by MD simulations.

By comparing these two CRSS for the magnitudes of fluctuation of 0 and $20 \%$, the CRSS is increased by the magnitude of fluctuation is increased. Therefore, the results suggest that the progress of the spinodal decomposition actually increase the CRSS, which must lead to a hardening of the material.The corresponding snapshots in Fig. 5 are indicated with a label of (a)-(d)
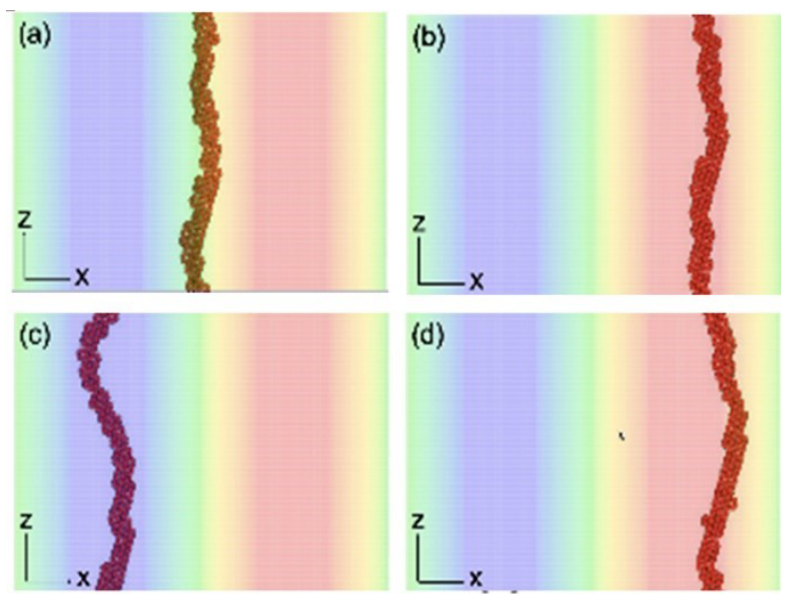

Fig. 5 Dislocation behavior in the MD simulation with a magnitude of $\mathrm{Cr}$ atom fluctuation of $20 \%$. Red particles show the atoms around the dislocation core.

Because the MD simulation is based on the interatomic potential, the MD simulations can account for all the mechanism given by the spinodal decomposition, such as elastic (internal stress) interaction and atomistic dislocation core influence. However, in the past studies, only the internal stress is taken into account. Thus, it is 
necessary to clarify which mechanism dominantly works in the material strength degeneration by the spinodal decomposition.

\subsection{Dislocation dynamics simulation results}

In order to reveal the mechanisms working in the material strength degeneration due to the spinodal decomposition, DD simulations in conjunction with the discretized PN model are performed. The necessary information for using the discretized PN model is the GSFE of the Fe-Cr alloy. The GSFE is calculated using the MD, and is shown in Fig. 6. The peaks of the GSFE of various $\mathrm{Cr}$ atom concentrations do not have any clear trend, and however, the maximum slope of the GSFE has a clear $\mathrm{Cr}$ atom concentration dependence, where the maximum slope becomes larger as the $\mathrm{Cr}$ atom concentration becomes lower.

Using the GSFE, we performed DD simulation of dislocation behavior in a spinodally decomposed $\mathrm{Fe}-\mathrm{Cr}$ alloy with the atomistic dislocation core influence. The snapshots of the DD simulation are shown in Fig. 7. The atomistic dislocation core influence is incorporated using the discretized PN model, and within the discretized PN model framework, the single dislocation is decomposed into 10 infinitesimal dislocations. In the figure, the color shows the assumed $\mathrm{Cr}$ atom concentration, where the red shows high, and the blue shows low $\mathrm{Cr}$ atom concentration.

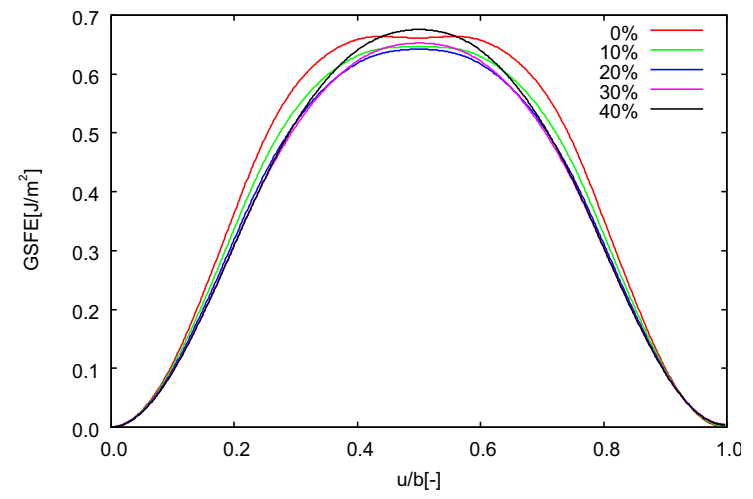

Fig. 6. Generalized stacking fault energy of Fe-Cr alloy with various $\mathrm{Cr}$ concentrations calculated using molecular dynamics method.

The dislocation core structure is slightly changed during the dislocation motion, particularly when the dislocation is located within the low $\mathrm{Cr}$ atom concentration. However, the dislocation shape keeps straight regardless the dislocation position. Along with the DD simulation with the atomistic dislocation core influence, the DD simulation without the atomistic dislocation core influence is also performed. In the simulation, the dislocation is described with a single dislocation line having the full Burgers vector. The snapshots of the simulation are shown in Fig. 8.
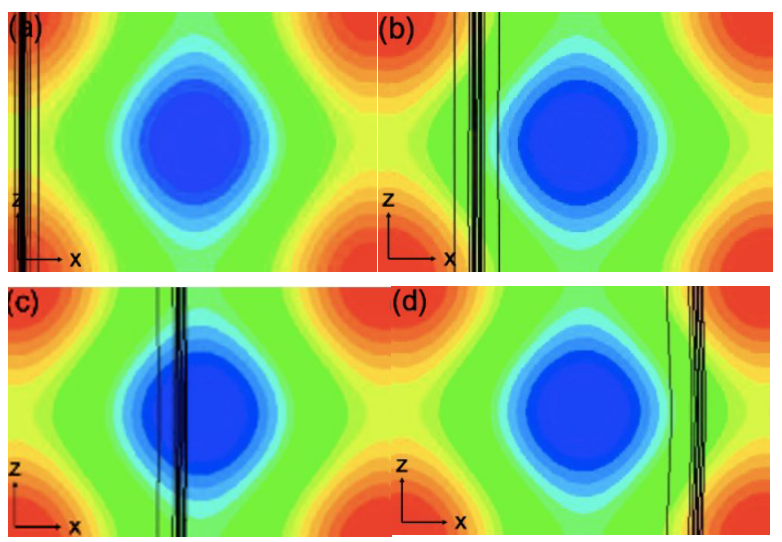

Fig.7. Dislocation behavior in spinodally decomposed $\mathrm{Fe}-\mathrm{Cr}$ alloy simulated using the DD method with the atomistic dislocation core influence.

Similar to Fig. 7, the shape of dislocation is not changed, and keeps straight. The CRSS calculated by the DD simulations are shown in Fig. 9 with those calculated by the MD simulations. In the results of MD simulations, there is a wide scatter for each magnitude of $\mathrm{Cr}$ atom fluctuation. In the MD simulation models, the $\mathrm{Cr}$ atoms are distributed using random numbers so that we performed the $10 \mathrm{MD}$ simulations with different simulation models created with different random seeds. In the figure, the CRSS is increased linearly as the magnitude of fluctuation is increased.

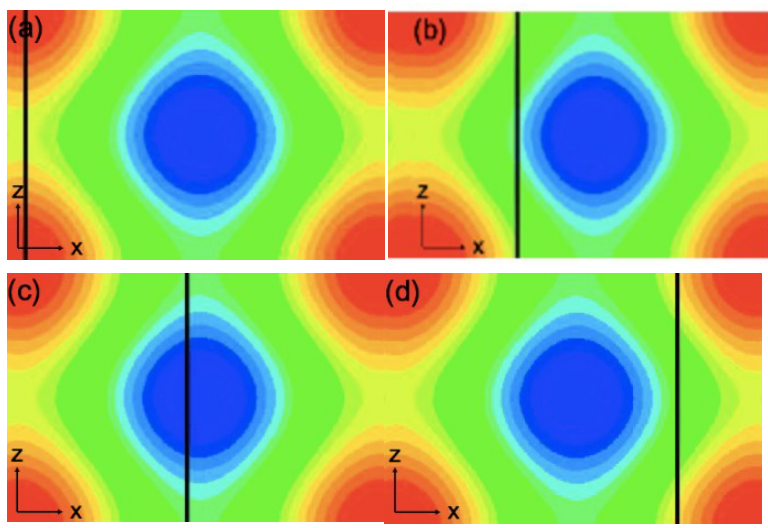

Fig.8. Dislocation behavior in spinodally decomposed $\mathrm{Fe}-\mathrm{Cr}$ alloy simulated using the DD method without the atomistic dislocation core influence.

The MD simulation results are almost identical to the DD simulation results. Interestingly, the CRSS calculated by the DD simulation with the atomistic dislocation core influence is the same as that calculated without the atomistic dislocation core influence, meaning that the atomistic dislocation core influence is negligible, and the internal stress is the dominant factor controlling the material strength of spinodally decomposed $\mathrm{Fe}-\mathrm{Cr}$ alloys. 


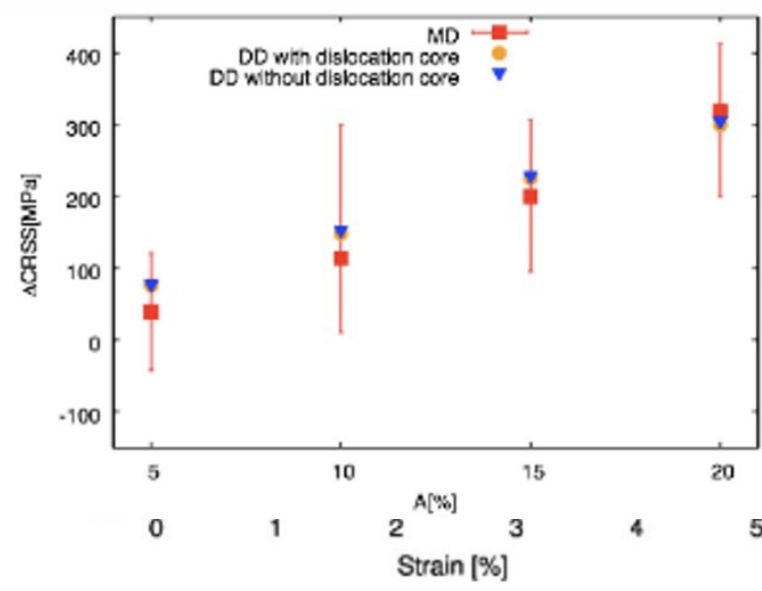

Fig. 9. Critical resolved shear stress (CRSS) for various magnitude of $\mathrm{Cr}$ atom fluctuation calculated by the MD and DD.

\section{Conclusions}

The dislocation behavior in spinodally decomposed Fe$\mathrm{Cr}$ alloy was simulated using the MD method. The $\mathrm{Cr}$ atom distribution was assumed using a simple cosine function, and the magnitude of $\mathrm{Cr}$ atom fluctuation was changed in a range from 0 to $20 \%$. The dislocation shape was extracted from the atomic volume using the CNA method. The CRSS could be calculated as the peak stress in the stress-strain behavior. In order to clarify the dominant mechanism controlling the material strength change due to the spinodal decomposition, the DD simulations with and without the atomistic dislocation core influence were performed. The atomistic dislocation core influence is incorporated into the DD simulations using the discrete PN model, while the internal stress is implemented using the classical equation for the internal stress. By comparing these simulation results of dislocation behavior and CRSS, the following summaries could be obtained.

- In the MD simulations, the dislocation motion is significantly affected by the internal stress distribution.

- The CRSS is increased linearly as the magnitude of $\mathrm{Cr}$ atom fluctuation is increased.

- In the DD simulation with atomistic dislocation core influence, the dislocation does not change the shape, meaning that the atomistic dislocation core influence can be negligible, and the internal stress is a dominant factor determining the material strength change due to the spinodal decomposition.

- The CRSS calculated by the MD and DD is almost identical.

These findings are very useful for simplifying the analysis of the mechanism of material strength change due to the spinodal decomposition. Particularly in the analysis using the DD simulations, the required computational effort for simulating the dislocation behavior is greatly reduced by taking into account only the internal stress without the atomistic dislocation core influence.

This study was supported by JSPS KAKENHI Grant Number JP16K05044.

\section{References}

[1] J. W. Cahn. Hardening by spinodal decomposition, ActaMetallurgica, 11 (1963) 1275-1282

[2] M. Kato. Hardening by spinodally modulated structure in b.c.c. alloys, ActaMetallurgica, 29 (1981) 79-87

[3] N.M.Ghoniem, L.Z. Sun. Fast-sum method for the elastic field of three-dimensional dislocation ensembles, Physical Review B, 60 (1999) 128140

[4] N.M.Ghoniem, S.H. Tong, L.Z. Sun. Parametric dislocation dynamics: A thermodynamics-based approach to investigations of mesoscopic plastic deformation, Physical Review B, 61 (2000) 914927

[5] J. El-Awady, S.B. Biner, N.M.Ghoniem. A selfconsistent boundary element, parametric dislocation dynamics formulation of plastic flow in finite volumes, Journal of the Mechanics and Physics of Solids, 56 (2008) 2019-2035

[6] A. Takahashi, N.M.Ghoniem. A computational method for dislocation-precipitate interaction, Journal of the Mechanics and Physics of Solids, 56 (2008) 1534-1553

[7] S. Banerjee, N.M.Ghoniem. G. Lu, N.Kioussis. Non-singular descriptions of dislocation cores: A hybrid ab-initio continuum approach, Philisophical Magazine, 87 (2007) 4131-4150

[8] A. Takahashi, N.M.Ghoniem. Structure of selfinterstitial atom clusters in iron and copper, Physical Review B,80 (2009) 174104

[9] G. Bonny, R.Pasianot, D.Terentyev, L.Malerba. Iron chromium potential to model high-chromium ferritic alloys, Philosophical Magazine, 91 (2011) 1724-1746 